INRA Prod. Anim., 2011, 24 (1), 145-156

\title{
Élevages et sociétés : les rôles multiples de l'élevage dans les pays tropicaux
}

\author{
V. ALARYI, G. DUTEURTRE2 , B. FAYE $3,4,5$ \\ ${ }^{1}$ CIRAD, ICARDA, 15 G. Radwan Ibn El-Tabib St., Giza, Egypt, P.O. Box 2416, Cairo, Egypte \\ ${ }^{2}$ CIRAD, DRASEC Direction Régionale du Cirad, Bureau 102, Bât 2G, Cité diplomatique de Van Phuc, \\ 298 Kim Ma, Hanoï, Vietnam \\ ${ }^{3}$ INRA, UMR0868 Systèmes d'Elevage Méditerranéens et Tropicaux, 2 place Viala, F-34060 Montpellier, France \\ ${ }^{4}$ CIRAD, Systèmes d'Elevage Méditerranéens et Tropicaux, Campus International de Baillarguet, \\ F-34398 Montpellier, France \\ 5 Supagro, Systèmes d'Elevage Méditerranéens et Tropicaux, 2 place Viala, F-34060 Montpellier, France \\ Courriel : veronique.alary@cirad.fr
}

Par sa faculté à se développer dans des espaces très diversifiés (du désert à la forêt tropicale) et parfois restreints (hors-sol) ou dans des milieux agroécologiques extrêmes (haute montagne, désert), l'élevage constitue une opportunité et une sécurité alimentaire et économique pour une grande diversité de groupes sociaux. Il est aussi un marqueur socioculturel de nombreuses sociétés du Sud.

Partout dans le monde, l'élevage a marqué de son empreinte les sociétés et les espaces. Dans les pays du Sud, zones à fortes contraintes climatiques, environnementales et socio-économiques, l'élevage et ses activités dérivées structurent probablement de façon plus marquée qu'ailleurs, à la fois la vie économique des ménages et des groupes de ménages, mais aussi la majorité des événements sociaux, culturels ou religieux.

Cependant, les changements à l'œuvre aujourd'hui (perturbations climatiques, accroissement de la pression démographique, urbanisation, intensification des productions, emprise du marché, internationalisation des échanges) induisent toute une série d'activités en lien avec l'élevage, et des changements notoires de l'activité d'élevage ellemême que ce soit dans les zones rurales ou périurbaines, voire urbaines (Steinfeld et al 2010). Plus encore que dans le passé (du fait des changements évoqués ci-dessus), et de façon plus marquée sans doute que dans les pays du Nord, l'élevage assure de multiples fonctions, promouvant à la fois des activités d'appoint pour assurer la couverture en produits alimentaires du ménage, la diversification des sources de revenus pour des besoins de trésorerie de la famille, la capitalisation des surplus sous forme d'épargne ou des activités de valorisation et de commercialisation des produits d'élevage (Duteurtre et Faye 2009).
Un des moyens d'aborder la diversité des rôles de l'élevage est de reconnaître sa multifonctionnalité. La multifonctionnalité de l'agriculture a été soulignée par divers auteurs notamment dans les pays du Nord (Collectif groupe Polanyi 2008) mais aussi dans quelques pays du Sud comme au Brésil (Bittencourt Machado 2009). Les rôles multiples de l'élevage ont cependant été insuffisamment analysés, notamment dans les pays du Sud, dans la perspective de la défense d'une activité soumise à bien des controverses (Steinfeld et al 2006). D'ailleurs, l'identification du rôle particulièrement important de l'élevage dans les défis alimentaires du siècle à venir (Delgado et al 1999) conduit à s'interroger sur les autres fonctions de ce secteur qui connaît de fait d'importantes mutations. Dans les pays du Sud, la spécificité de ces fonctions multiples tient moins à l'activité elle-même, quasi identique dans ses fondements à celle mise en œuvre au Nord, qu'aux contextes sociaux, culturels, économiques et climatiques qui en révèlent tout le poids dans la société.

Nous aborderons dans cette synthèse successivement les fonctions environnementales, sociales et économiques de l'élevage, suivant en cela la typologie des fonctions multiples de l'agriculture proposée par Jean (2007 cité par Bittencourt Machado 2009). Les fonctions environnementales de l'élevage ne se réduisent pas à ses externalités positives ou négatives, mais concernent sur- tout les capacités d'adaptation de 1'activité d'élevage à des conditions difficiles. Les fonctions sociales sont considérées en tenant compte des contributions à la fois aux champs culturel et social des activités d'élevage. Enfin, les fonctions économiques doivent être abordées aussi bien dans l'économie des ménages que dans l'économie locale ou régionale, voire nationale.

\section{1 / L'élevage comme activité d'adaptation à des condi- tions difficiles}

\section{1 / Adaptation aux conditions climatiques et environnemen- tales}

L'élevage occupe une grande diversité de milieux écologiques, agronomiques et humains, bien qu'il reste l'activité dominante des environnements difficiles, impropres aux cultures, en raison: i) des conditions climatiques telles que les températures extrêmes ou les écarts de températures jour/nuit, le niveau d'aridité ou d'humidité, l'occurrence des sécheresses, ii) des contraintes pédologiques (profondeur des sols, fertilité, texture, hydrologie, contraintes chimiques comme la salinité...) ou physiques telles qu'un relief accidenté. Les conditions d'aridité concernent plus des trois quarts des terres d'Afrique du Nord, d'Asie centrale et d'Ouest et plus 
de la moitié des terres d'Afrique de l'Ouest et du Sud. Pour tous ces milieux, un facteur commun de survie et de développement socio-économique est bien l'élevage en tant que capital sur pieds et source d'échanges et de revenus pour favoriser la vie sociale.

Le développement et le maintien des activités d'élevage dans ces milieux extrêmes sont principalement basés sur les mécanismes de résistance biologiques de certaines espèces ou races (stockage d'eau chez les dromadaires, réserves énergétiques caudales chez les brebis Barbarine de Tunisie) ou la capacité à valoriser de multiples espèces végétales (Rutagwenda et al 1989). Les petits ruminants et dromadaires dominent largement les zones arides de l'Asie de l'Ouest, d'Afrique du Nord et du Nord Sahel (qui s'étend du NordOuest de la Mauritanie au Nord-Est du Tchad). Les camélidés offrent des opportunités d'activités aux limites du désert pour les dromadaires et chameaux, ou aux plus hautes altitudes pour les lamas et alpagas.

Dans ces zones, la mobilité a constitué un élément quasiment permanent d'adaptation aux contraintes du milieu et de gestion des ressources. Elle s'inscrit autant dans l'itinérance à la recherche de nouveaux parcours, la gestion d'une soudure entre bonne et mauvaise année, que dans les circuits de commercialisation qui se construisent sur des réseaux sociaux. Aujourd'hui, la mobilité de longue durée tend à se restreindre accompagnant le déclin du système nomade ou transhumant au profit de systèmes agropastoraux jusqu'aux confins des zones arides. Les raisons sont multiples en lien avec des facteurs externes (crises climatiques ou politiques qui ont obligé les éleveurs à décapitaliser puis se diversifier vers l'agriculture (Arditi 2009), politiques de sédentarisation plus ou moins forcées), et internes liés à la modernisation, aux aspirations des jeunes générations et à l'émancipation des dépendants (Alary et El Mourid 2005). Ces systèmes agropastoraux sont multiples selon les milieux sociogéographiques et les conditions agroclimatiques qui conditionnent l'importance des mécanismes de mobilité. Il existe ainsi un gradient depuis les systèmes semi-nomades et transhumants (comme certains groupes Peuls en Afrique subsaharienne, les Bédouins en Asie occidentale, ou les Bahimas en Ouganda...) aux systèmes sédentaires ou encore systèmes mixtes agriculture-élevage qui englobent à la fois les cultivateurs qui investissent dans l'élevage pour conserver leur épargne et les éleveurs qui développent un système de culture, soit pour l'autoconsommation, soit pour la vente (cultures

Photo 1. Elevage de petits ruminants dans la New Valley (Egypte).

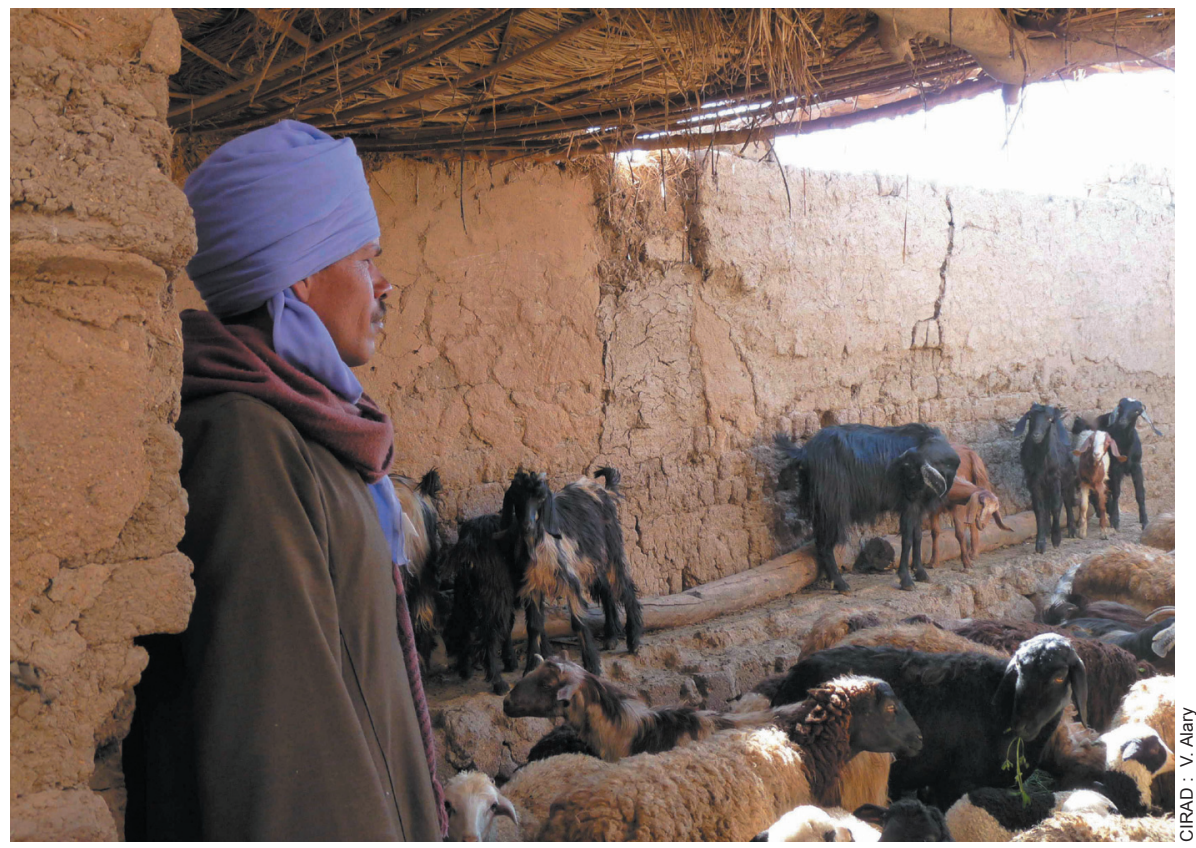

de rente) dans les zones pluviales. Les principales différences entre ces deux grands systèmes concernent à la fois la taille du troupeau et le mode de gestion des ressources. Si les semi-nomades et transhumants restent très dépendants des ressources collectives, les systèmes mixtes et agropastoraux sont extrêmement dépendants du foncier approprié.

Le développement du système agropastoral se traduit par une augmentation de l'utilisation des résidus de récolte dans l'alimentation animale, des céréales fourragères et d'autres concentrés à travers la vaste zone dite semi-aride $(100-400 \mathrm{~mm}$ de précipitations annuelles) qui s'étend du Maroc à la Mongolie (Nordblom et al 1997), mais aussi dans les pays d'Afrique subsaharienne (Corniaux et al 1999). Les cultures fourragères (orge, berseem, sorgho, vesce, avoine) sont limitées aux zones irriguées. La majorité des agropasteurs développe la production de céréales sur des terres marginales dont les rendements restent très dépendants des aléas climatiques. En Afrique du Nord, les ressources pastorales ne couvriraient plus que $10 \%$ des besoins (Nefzaoui et El Mourid 2008), ce qui constitue un facteur de dépendance accrue à la fois au marché mais aussi aux aides publiques en cas de sécheresse et donc une fragilisation des systèmes durant les mauvaises années climatiques

Ainsi, l'élevage peut être considéré comme un marqueur géographique de nombreuses zones impropres à l'agriculture, comme on peut l'observer dans les zones sahéliennes ou les zones steppiques du Maroc à la Mongolie selon un gradient Ouest/Est. Cette frange marquée par l'aridité est souvent le domai- ne de sociétés dites d'éleveurs, dans le sens où l'activité d'élevage est au cœur de la structuration et l'organisation sociopolitique et spatiale des populations.

\section{2 / Moyen de survie ou de déve- loppement économique face aux changements globaux}

Dès que les conditions agronomiques s'améliorent, se développent des activités liées à l'élevage bovin et bubalin que l'on retrouve dans une bonne partie de l'Amérique du Sud, l'Afrique subsaharienne soudanaise et l'Asie du Sud. En Asie de l'Est, sous la pression démographique et foncière, le système d'élevage dominant combine généralement des espèces à cycle court telles que les porcins et les volailles. En Asie du Sud (Inde) ou dans la vallée du Nil (Egypte), on trouve des systèmes d'élevage de ruminants en quasi hors-sol, utilisant peu de place, très proches finalement du modèle de basse-cour (BravoBaumann 2000) où les animaux sont élevés dans la cour de la maison avec un modèle d'alimentation à l'auge.

Aujourd'hui, avec la segmentation des territoires (par les frontières) notamment en Afrique, les migrations de colonisation se sont plus ou moins taries laissant la place à des migrations économiques déterminées par la pauvreté et l'exclusion (Auclair et al 2001). Cette migration a changé de contour : elle prend un caractère plus individuel et familial que collectif, elle se complexifie sous l'effet de flux de réversibilité. Elle s'accompagne de profonds changements des milieux, 
notamment la conversion des espaces forestiers et des savanes en terres agricoles, avec ses conséquences directes sur l'érosion des sols, la déforestation, l'appauvrissement des terres. Elle s'accompagne aussi d'une évolution des sociétés : changements de mode de vie, mutations sociales et familiales. Dans ces mutations, on observe le rôle actif de l'élevage dans l'avancée des fronts pionniers, notamment grâce à l'utilisation de la force animale (traction attelée). Cette activité est certes souvent décriée pour son impact dans la déforestation et la substitution du couvert forestier par les pâturages. Toutefois, ces dynamiques environnementales peuvent être également lues comme des constructions paysagères nouvelles, dans lesquelles il s'agit moins de vilipender l'élevage que de trouver un nouvel équilibre entre les différents agroécosystèmes (Bommel et al 2010). Ainsi, en Amazonie, l'élevage peut être aussi vu comme un moyen d'anthropiser des espaces forestiers, jusqu'alors peu fréquentés par l'Homme, en ouvrant la voie au développement agricole (Ickowicz et al 2010).

L'élevage joue aussi un rôle essentiel dans les systèmes agricoles pour le maintien de la fertilité des sols. On citera, à titre d'exemple, les étables fumières du Sénégal ou du Burundi où la production de lait devient (en termes de revenu et d'intérêt agronomique) un produit secondaire par rapport au fumier, principal produit pour le développement des cultures vivrières intensives dans des régions rurales à forte densité.

Parfois grâce à ses produits, ses modes de production et les formes d'organisation sociale associées, l'élevage peut devenir un marqueur du territoire en générant une succession d'activités économiques, comme cela est par exemple observé dans certaines zones où la production laitière s'est fortement développée. On peut citer le cas du Sénégal (Sow-Dia et al 2007) ou du Pérou (Aubron 2007). L'activité laitière induit aussi un ensemble d'activités sociales et économiques en amont (matériel de traite, intrants vétérinaires et aliments pour bétail...) et en aval (réseaux de transformation et de distribution, outils de valorisation...), qui en font un moteur du développement local.

De fait, l'élevage ne se cantonne pas aux zones difficiles bien qu'il en soit la principale activité quand les conditions climatiques ou pédologiques ne permettent plus de cultiver. Toutefois, l'élevage grâce à sa mobilité et ses capacités d'adaptation aux conditions climatiques difficiles constitue une activité essentielle de survie, voire de développement. Ainsi l'élevage s'inscrit comme un moyen d'adaptation des sociétés aux conditions du milieu et aux changements extérieurs. Il se situe à l'interface entre la société et la gestion du milieu, répondant directement aux changements du milieu et induisant également des transformations des écosystèmes (en partant ou en arrivant).

\section{2 / Les troupeaux : entre contribution culturelle et capital social}

\section{1 / Les dimensions sociales, culturelles et symboliques des animaux}

De nombreux travaux en anthropologie et sociologie ont mis en évidence différentes contributions de l'élevage à la structuration politique et l'organisation socioculturelle des sociétés d'éleveurs, notamment en Afrique subsaharienne (voir par exemple Bernus 1975, 1981 et Bonte 2007, 2008). Certains travaux s'intéressent aux modes de vies des éleveurs et mettent en exergue certains traits relatifs à l'habitat, au territoire, aux techniques d'élevage, comme aux fonctions symboliques du bétail. D'autres insistent davantage sur l'organisation socio-institutionnelle de ces sociétés et sur les règles qui structurent à la fois leur mode de vie (gestion de l'espace et des ressources, sécurisation d'un habitat) et sa reproduction (système matrimonial, identité symbolique). Si l'approche culturelle des sociétés d'éleveurs basées sur le nomadisme, en opposition aux sociétés sédentaires, fait toujours débat en matière de facteur de différenciation identitaire (Bonte 2006), le système symbolique existant autour de l'élevage (Bernus 1984), qui unit et différencie les différentes communautés d'éleveurs, révèle bien le rôle culturel de l'élevage. Ce système symbolique se retrouve dans de nombreux domaines tels que les règles matrimoniales, les rapports économiques, le langage ou la religion et l'animal se trouve enchâssé dans des règles sociales complexes. Ainsi, au-delà de sa valeur économique, le capital cheptel constitue à la fois un héritage culturel et un des piliers de l'organisation sociale des groupes de pasteurs.

A titre d'exemple, on peut citer la pratique du préhéritage commune à bien des peuples pastoraux (Dupire 1970) conférant au jeune pasteur un réel statut social et économique ou le rôle de l'élevage dans le système de régulation sociale de la dot et de la dette agissant comme facteur de redistribution (Faye 2009). L'élevage est souvent au centre des alliances matrimoniales : chez les Peuls Wodaabés, le père du marié donne une génisse au père de la mariée alors que l'époux donne à sa nouvelle femme des animaux dont elle garde l'usage et dont le capital est réservé aux enfants du couple (Thébaud 1988). On voit ainsi comment l'animal participe à la constitution de chaînes de relations sociales verticales (intergénérationnelles) et horizontales (entre familles d'une même tribu ou clan), ou à des mécanismes de redistribution qui participent à la sécurité présente et future du groupe.

Le rôle important des animaux dans les relations sociales et dans nombres de cérémonies, rites ou pratiques ostentatoires est un élément commun aux sociétés d'éleveurs, mais aussi à de nombreux groupes d'agro-éleveurs ou d'agriculteurs. Dans les systèmes agricoles ou agropastoraux, l'élevage peut faire l'objet de nombreux échanges entre membres des communautés et est susceptible d'acquérir une dimension sociale, symbolique, ou un rôle de marqueur culturel. L'élevage participe aussi de manière centrale aux trajectoires d'évolution des familles sahéliennes, qui peuvent prendre la forme «d'allers et retours» entre pastoralisme et agropastoralisme, en fonction de l'état de capitalisation des familles (Bonfiglioli 1990). Enfin, l'élevage joue un rôle important dans les trajectoires d'accumulation des paysans des fronts pionniers amazoniens et il acquiert, à ce titre, un statut particulier au sein de ces communautés agricoles (Tourrand 2009).

Ce «marquage» culturel par l'élevage apparaît aussi au sein même des sociétés urbaines. En Inde, les produits laitiers s'inscrivent dans un système symbolique multiple combinant la religion (par le biais du symbole de la vache «sacrée»), le système matrimonial (par le système de la dot) et la réussite économique nationale au travers de la coopérative laitière, National Development Dairy Board (NDDB) (Alary 1999). Dans les villes sahéliennes, les animaux domestiques peuvent se révéler être de puissants facteurs d'identité pour les populations d'origine pastorale récemment urbanisées, mais aussi pour certains individus à la recherche d'une nouvelle identité et d'une reconnaissance sociale. Cette importance culturelle s'illustre par exemple avec le développement de l'élevage de mouton de prestige dans les arrière-cours de Dakar (Ninot 2010). Elle s'illustre aussi dans les modes de consommation urbains qui laissent une large part aux préparations «traditionnelles» à base de produits laitiers et de produits carnés, notamment 
pour les fêtes (Duteurtre 2003, Brisebarre et Kuczynski 2009).

\section{2 / Élevage et capital social}

Le capital social constitue un maillon essentiel et critique des stratégies de lutte contre la pauvreté et de développement économique durable. Le capital social est défini comme l'ensemble des normes et réseaux, et la capacité des gens à agir collectivement (Woolcock et Narayan 2000). Il est considéré dans l'approche des «capabilités» formalisée par Sen (1992) comme un élément essentiel de la réduction de la vulnérabilité.

Cependant, le capital social fait l'objet de nombreux débats sur sa définition et ses retombées. Pour Fafchamp et Mintens (2002), il peut être défini comme : «a stock of emotional attachment to a group or society at large that facilitate the provision of public goods [...]»; pour Putman (1993), il s'agit principalement d'une externalité collective et donc d'un bien public ; alors que Coleman (1988) suppose que le capital social et donc ses intérêts (revenus du capital) sont individuels même s'il résulte d'une interaction sociale. Pour Fafchamps (ibid.), les bénéfices du capital sont principalement issus de la réduction des coûts de transaction alors que, dans la conception beckerienne, les revenus de cet actif se matérialisent soit comme un flux d'utilité perçu par des individus, soit comme une augmentation des rendements d'une fonction de production (RéquierDesjardins 2003).

Cette notion de capital social est particulièrement importante dans le contexte africain où les liens tissés constituent à la fois un facteur de sécurité (entraide en situation difficile, diminution des risques par la possibilité de mener, au niveau d'un groupe, des stratégies diversifiées), et un facteur d'optimisation des systèmes de production par l'organisation de synergies, comme la régulation de l'accès aux ressources dans les sociétés d'élevage pastoral. Le capital social peut aussi être un facteur de domination, de dépendance, de fragilité, voire un facteur d'exclusion ou de marginalisation. Il est communément admis en Afrique subsaharienne que la famille élargie exerce, par le jeu des solidarités, une fonction de redistribution des richesses, mais aussi un rôle de partage des charges d'une descendance nombreuse (Mahieu 1989, Adjamagbo 1997), qui conduit souvent à des situations de dominance, de dépendance et de fragilité. Ainsi, les interactions sociales sont des processus complexes avec des effets négatifs (inhibition de l'initiative privée, faible incitation à accroître les gains du fait de la pression sociale, déresponsabilisation, relation de dépendance, voire domination) comme des effets positifs (prise en charge familiale d'une protection sociale non assurée par l'économie publique).

L'une des formes les plus connues de capital social propre aux systèmes d'élevage sont les réseaux de confiage et de redistribution du cheptel. Le don ou le confiage de la vache ou de tout autre animal «de rente» est une pratique classique dans la plupart des sociétés pastorales. Si le don est formellement un mécanisme d'aide au pauvre, à celui qui a perdu tout ou partie de son bétail au point qu'il ne peut plus en vivre, le confiage est avant tout un élément d'une stratégie de prudence par la dispersion

\section{Photo 2. Marché de Roro au Sud-Est du Tchad.}

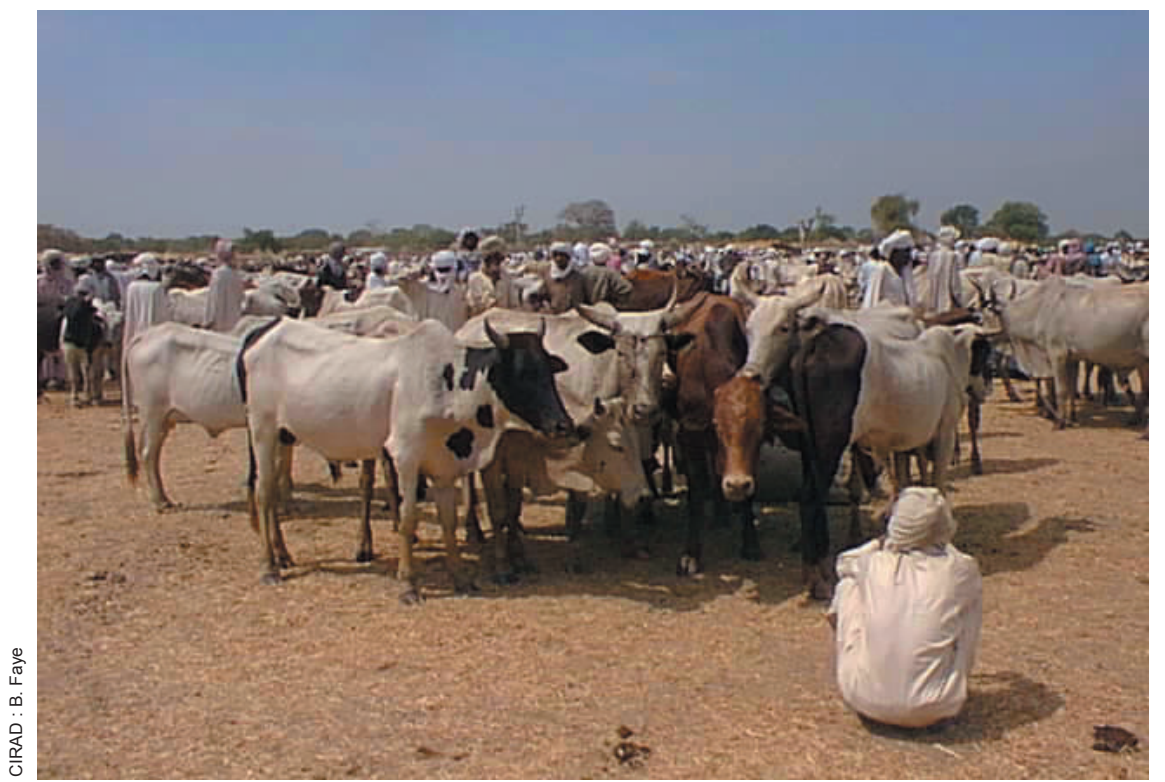

géographique et sociale du cheptel sur la base d'une relation contractualisée (Faye 2009). Il participe aussi à un mécanisme de solidarité au sein de la communauté pastorale qui implique une certaine réciprocité en cas de besoin ; tout animal confié est susceptible de revenir dans son troupeau d'origine en cas de difficulté du propriétaire (sécheresse, épizooties, dépense importante).

Le transfert d'animaux par dons ou prêts peut prendre des formes très diverses, variant d'un groupe ethnique à l'autre. Chez les Peuls Wodâabe (Niger), "la vache habbanae (le "contrat» associé est alors appelé habbanaaji) est celle qui est prêtée temporairement à un parent ou un ami jusqu'à ce qu'elle ait mis bas trois fois, les produits devenant la propriété de l'emprunteur. La vache fewnaange est prêtée à un ami qui vient de perdre un animal. Le confiage pour une durée indéterminée à un cousin croisé est appelé soggarae. Lorsqu'il s'agit d'une vache prêtée à une famille temporairement privée de lait, on parle de vache diilae. Le transfert d'un mâle reproducteur pour répondre aux besoins de reproduction d'un troupeau qui en est démuni peut également avoir lieu auquel cas l'emprunteur est généralement tenu de rendre l'animal au bout de quelques temps à son propriétaire avec une génisse. Ces transferts diffèrent fondamentalement du gardiennage par les bergers de vaches jokereeji, c'est-à-dire d'animaux appartenant à des étrangers (commerçants ou fonctionnaires). En effet, pour ces bergers, le lait représente une rémunération» (Faye 2009). Dans la plupart des cas, ces transferts concernent des vaches ou d'autres animaux, en âge de se reproduire, confirmant le rôle de sécurisation que jouent ces mécanismes. Les transferts peuvent aussi être temporaires et ne concerner alors que les produits (naissances et/ou produits tels que le lait).

Le confiage des animaux à un ensemble de parents, de relations ou de membres du clan contribue à la constitution d'un «réseau social de solidarité» (Gallais 1989). Le prêt et le don sont des éléments permettant de tisser des alliances, d'entretenir des liens de parenté et d'amitié. Ils contribuent surtout à la viabilité des unités domestiques temporairement non viables parce qu'affectées par une surmortalité de leur bétail (sécheresse, épizootie) ou par un déstockage involontaire ou contraint. Ils sont aussi le moyen pour un éleveur dépassant sa capacité de main-d'œuvre de favoriser la circulation de l'excédent du troupeau en répartissant les charges animales dans l'espace. Au fond, ce qui confère du prestige au sein de ce réseau social, c'est moins la possession de richesses en bétail 
que sa distribution (Levi-Strauss 1967). Derrière l'avantage «numérique» de disposer d'un grand nombre d'animaux, il y a l'intérêt d'avoir un grand nombre d'amis et de relations (Waller 1999). La redistribution du bétail génère un réseau «d'obligés». Une telle construction sociale n'est pas envisageable avec une richesse monétaire. Chez les peuples pasteurs, l'argent ne peut être le véhicule des relations sociales et identitaires et en aucun cas ne peut entrer dans des mécanismes de redistribution.

Cependant, les stratégies de redéploiement du capital animal n'ont pas empêché les risques d'épuisements biologiques au cours des récentes sécheresses. La surmortalité du bétail, la détérioration des termes de l'échange pastoral (augmentation du prix des céréales et chute du prix du bétail en période de sécheresse), la péjoration des ressources en fourrages et en eau, la désorganisation des écosystèmes concourent parfois à dépasser la capacité de réponse du système basé sur les réseaux de solidarité. Ainsi, les crises démographiques, dont ont été victimes les troupeaux suite aux récentes sécheresses, déstabilisant le système traditionnel d'assistance mutuelle, se sont traduites par des réorientations de l'activité (vers l'agriculture notamment) ou par l'exil vers les centres urbains, poussant chacun sur des positionnements plus individualistes, incompatibles avec la répartition du capital. Dans les cas extrêmes de paupérisation généralisée, la mutualisation du risque conduit la redistribution non pas à faciliter la reconstitution du cheptel des plus démunis, mais simplement à répondre à la survie immédiate, ce qui se traduit par l'échange de l'animal donné ou confié contre des grains, voire à l'autoconsommation de l'animal, abolissant par là même toute possibilité de réciprocité (Anderson 1999). Dès lors, ces mécanismes de solidarité ne suffisent plus à sortir les pasteurs de la marginalisation collective. Ils peuvent aussi éroder sérieusement la capacité de réponse du système à des stress externes.

Dans les sociétés agropastorales d'Afrique du Nord, l'individualisme, par érosion du capital social, explique en grande partie les difficultés de gestion commune des ressources renouvelables, comme les parcours, et l'adoption de technologies susceptibles de favoriser un développement durable (Dutilly-Diane et al 2005). En effet, les technologies relatives à la durabilité des ressources naturelles (plantations, mise en défens...) s'inscrivent généralement dans le temps et nécessitent une prise en charge par une communauté d'individus du fait de l'insuffisance de l'épargne pour supporter l'investissement. Les conséquences de l'absence de capital social conduit alors les individus à privilégier les investissements à retour rapide et de montant peu élevé, susceptibles d'éroder le capital naturel, ou encore à se réfugier dans des stratégies défensives (logique extensive, statu quo technique) qui sont conjointes à des comportements opportunistes sur les ressources naturelles. De plus, l'innovation individuelle accroît les risques: risque technique lié à la phase de transition du système productif et risque social lié aux jalousies suscitées.

A l'instar des autres activités de diversification et à l'interface entre le social et l'économique, l'élevage porte aussi en soi le risque d'un affaissement global de la formation et d'une marginalisation croissante d'une certaine classe sociale par le jeu de la déscolarisation, comme il a été observé en Inde pour les enfants et plus particulièrement les filles (Alary 2009). Ainsi l'organisation socioculturelle autour de l'activité d'élevage n'est pas neutre dans ses effets, en termes de capital humain et donc de réduction de la vulnérabilité. Cette organisation sociale fortement imbriquée autour de l'activité d'élevage se retrouve à l'échelle des ménages ou des familles où se concrétisent les phénomènes de pauvreté ou d'insécurité alimentaire.

\section{3 / Les fonctions éco- nomiques de l'élevage à l'échelle des familles}

\section{1 / Entre trésorerie (activité productive) et sécurité (capital sur pieds) : des réalités qui se recouvrent au sein d'un même élevage}

L'élevage constitue par essence un objet complexe; principal mécanisme d'accumulation et de transmission de richesse dans les sociétés pastorales et agropastorales, principal mécanisme d'épargne dans les sociétés rurales du Sud, il reste toujours un capital productif plus ou moins actif selon les besoins courants du ménage ou les événements familiaux ou sociaux - qui peuvent générer des dépenses ostentatoires - ou les risques extérieurs (crise climatique, spéculation sur les marchés...). Les produits sont multiples avec des pas de temps productifs très variés ce qui rend la complémentarité des espèces très sécuritaires. On peut citer des produits intermédiaires comme le lait, les œufs, la traction animale ou le transport, la fumure et des produits finaux comme les peaux et cuirs et la viande... ou encore la combinaison des espèces à cycles courts (volaille, porc), bisannuels (petits ruminants) ou pluriannuels (ruminants).

Comprendre le rôle de l'élevage dans les exploitations revient à attribuer un poids à ces différentes fonctions entre production et épargne et donc aux formes d'exploitation et de valorisation des produits de l'élevage, reflétées dans les pratiques d'élevage. Généralement la fonction d'épargne se révèle en partie dans le croît net du troupeau et sa structure démographique, alors que le degré d'investissement dépend du taux d'exploitation (incluant les achats et ventes d'animaux). Les fonctions de trésorerie sont mises en évidence par l'exploitation des produits intermédiaires (lait, laine, mise en location, transport) ou la vente régulière de certains types ou catégories d'animaux.

Les études typologiques des systèmes fonctionnels d'exploitation que ce soit dans des sociétés agropastorales ou rurales mettent de façon «quasi naturelle» en évidence le rôle différencié de l'élevage selon un gradient allant des fonctions de trésorerie aux fonctions de capitalisation et d'épargne. Ce rôle varie selon l'histoire et le stade de vie du ménage (selon l'approche des cycles de vie de Tchayanov 1924), le degré et le type de diversification des activités au sein du ménage (en différenciant les activités d'élevage, des activités culturales vivrières ou de rente et de la nature des activités non agricoles : activité salariale ou privée...) ou bien les événements conjoncturels qui peuvent faire varier brutalement le cursus. C'est ainsi que dans des études sur les systèmes laitiers en Inde ou en Ouganda, on retrouve des rôles similaires d'épargne ou de valorisation des produits laitiers en fonction des formes de diversification et de l'âge du chef de ménage (Alary et al 2007, Alary 2009). Mais l'identification de ces rôles différenciés ne doit pas masquer le rôle potentiel des autres fonctions qui varient selon les espèces.

Ces différents rôles sont bien révélés dans les approches en termes de moyens d'existence (Livelihood approach) (Ellis et Mdoe 2003, Carter et Barrett 2006). Ces approches s'inscrivent en partie sur les théories du bien-être proposées et formalisées par Sen (2002) qui a mis l'accent sur les «capabilités» des agents comme moyen de réduire leur vulnérabilité. Ainsi, une approche des niveaux de richesses dans les systèmes oasiens de la New Valley en Egypte, basée sur les dotations en capital des ménages classés selon des groupes de pauvreté monétaire, montre comment l'élevage de petits ruminants peut être un moyen de sortie de la grande pauvreté (figure 1). 
Figure 1. Profils de composition du capital en fonction du revenu moyen per capita des ménages (Source : données d'enquête, New Valley, Egypte, 2010).

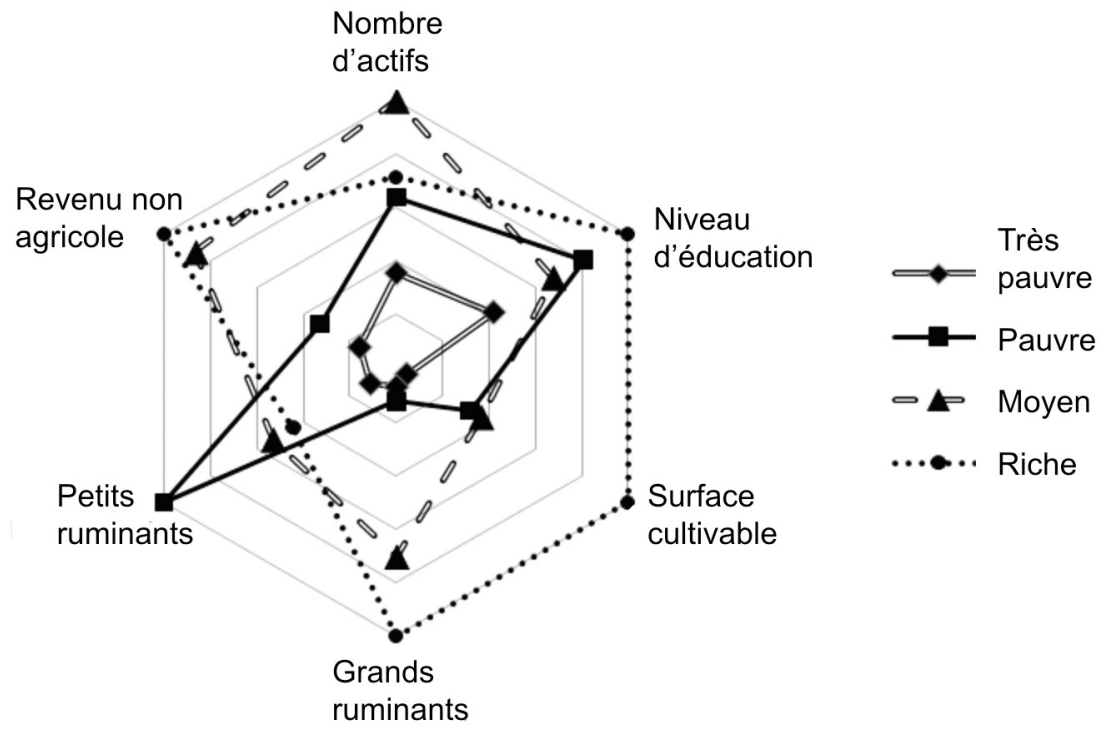

\section{2 / Rôle différencié et complé- mentaire des espèces animales au sein d'un ménage}

Dans les régions du Sud, il est fréquent de rencontrer des élevages mixtes dans les exploitations où chaque espèce peut jouer un rôle dominant particulier. Dans la majorité des zones arides d'Afrique du Nord et du Nord du Sahel, les élevages sont composés généralement d'ovins, caprins, voire de camelins, permettant ainsi une répartition des risques (épidémiologiques, d'accès aux ressources, de sensibilité aux aléas climatiques) entre les espèces (Faye 1992). Si les ovins sont principalement destinés à la vente sur les marchés, les caprins sont souvent associés aux gestes traditionnels de convivialité au sein de la famille élargie ; ils permettent aussi d'assurer un certain statut social (capacité à bien «recevoir» ses hôtes) durant les fêtes familiales ou religieuses. Les dromadaires représentent le principal capital sécuritaire de la famille en cas de grosses dépenses imprévues, mais aussi un moyen de déplacement et donc d'échange non négligeable. Dans les zones montagneuses des Andes, Tichit décrit comment la viabilité des exploitations dépend des fonctions complémentaires de chaque espèce au sein du troupeau (Tichit et al 2004). Dans les régions des Cerrados au Brésil où se développe une activité laitière intensive, nombre de producteurs maintiennent dans la phase transitoire de constitution du cheptel laitier des vaches zébus pour la viande ; le maintien d'un élevage porcin pour l'autoconsommation concerne tous les ménages. Dans les régions agricoles irriguées ou non d'Afrique, il n'est pas rare de voir se développer une activité d'élevage complexe, associant des petits ruminants (ovin, caprin) pour satisfaire les dépenses courantes ou saisonnières du ménage et des gros ruminants (bovin ou bubalin) qui constituent à la fois la sécurité financière du ménage ainsi que sa sécurité alimentaire via l'approvisionnement en lait de la famille (Corniaux et al 2009). Dans les zones plus densément peuplées, notamment en Asie, la combinaison se fait autour des élevages à cycles courts (élevages porcin et avicole).

La diversification des espèces au sein des exploitations dans les pays du Sud est complexe et répond à une multitude de facteurs à la fois agronomiques et sociaux en lien avec la gestion de l'espace et des ressources (Gibon 1997), économiques (satisfaction des besoins de trésorerie ou d'emploi), financiers (sécurité financière, absence de système bancaire), nutritionnels (complément protéinique) et socioculturels (satisfaction des gestes de convivialité qui assurent une certaine reconnaissance et participent à la construction d'un capital social). Dans certaines économies familiales, il peut y avoir des appropriations multiples du troupeau ou une combinaison entre propriétaires et usagers ; les femmes ou les personnes dépendantes peuvent notamment démarrer un petit élevage qui va leur permettre de couvrir soit leurs dépenses personnelles (amélioration du bien-être individuel) soit servir d'assurance face à un chef de ménage imprudent (assurance collective). En Afrique, il n'est pas rare que les femmes soient aussi propriétaires de quelques têtes de petits ruminants qui leur permettent de couvrir des dépenses alimentaires ou de scolarité pour leurs enfants. On a vu précédemment, que les femmes peuvent recevoir en dot des ani- maux dont elles ont l'usage et la propriété temporaire jusqu'aux descendants.

A ces systèmes, on pourrait attribuer «la notion de système d'activités plurispatiales» telle que développée par Lesourd (1997). Ainsi à l'unité de production se substitue le ménage comme unité familiale globale, multi-active et plurilocalisée pour aborder les rôles complémentaires de chaque espèce dans la viabilité du ménage.

\section{3 / L'élevage dans la diversifi- cation des activités et la sécurisa- tion des revenus}

L'élevage joue aussi un rôle moteur ou accélérateur de la diversification dans le secteur agricole ou non agricole. Ce degré de diversification est particulièrement important dans les zones très vulnérables (forte variabilité climatique, baisse de fertilité des sols) comme les zones où se développe une activité commerciale importante grâce au dynamisme agricole (Reardon et al 1992). Mais ce rôle de diversification varie en fonction du nombre de travailleurs dans les ménages, des revenus issus de la principale activité et bien sûr des opportunités ou options dans une zone donnée ou des possibilités d'émigrer.

Tout d'abord la diversification des activités animales ne répond pas toujours aux mêmes objectifs ou critères de choix que la diversification sous forme d'activités culturales ou de travail de salarié agricole, par exemple. Généralement, l'acquisition des premiers animaux répond souvent à une forme de thésaurisation de la vente du surplus agricole et c'est souvent une fois atteint une certaine taille, que le troupeau devient alors une activité de diversification par la valorisation des produits animaux (veaux, lait, fumier) et donc un apport de revenu monétaire régulier. Il s'agirait donc plus du produit ou résultat de la diversification des sources de revenu, pour ensuite devenir un facteur de contribution à cette diversification ainsi qu'un facteur d'intensification agricole (grâce à l'énergie et la fertilisation organique fournies par les animaux). Ceci est surtout vrai dans les communautés agricoles qui démarrent une activité d'élevage, et ce à la différence des sociétés agricoles qui ont toujours pratiqué simultanément l'agriculture et l'élevage (cas des exploitations indiennes, ou exploitations appelées «mixed farming systems» en Afrique de l'Est).

L'élevage peut être aussi au centre d'une diversification. Boutrais (1994) montre comment les Foulbé de l'Adamaoua dans le Cameroun septentrional ont su s'adonner à l'agriculture dans les périodes de crises sanitaires 
(perte de bétail suite à la trypanosomiase bovine) ainsi que face aux changements sociaux et politiques (libération des anciens esclaves en charge des travaux agricoles chez les Foulbé). De plus, les Foulbé ont de longue date su associer le commerce de bétail à l'élevage. Si certains ont su agrandir leur troupeau grâce au bénéfice du commerce, d'autres ont été contraints de revenir au pastoralisme lorsque les affaires ont décliné. Le convoyage des animaux, notamment vers le Sud du Cameroun, constitue aussi une activité très attractive pour les jeunes, qui tentent parfois leur chance dans le commerce parallèle de petits objets, de noix de cola, de commerce de vêtement, etc. Ainsi, il existe des liens étroits entre élevage et commerce : la vente de bétail permet de démarrer un commerce ou une boutique. Les activités religieuses, telles que maîtres d'école coranique ou écrivains de versets de prière, sont aussi un moyen de se constituer un troupeau du fait que la majorité des récompenses consistent en bétail. Ainsi, l'élevage peut se conjuguer à de nombreuses activités et le champ est d'autant plus large que les animaux sont un excellent moyen d'exploiter la diversité spatiale. Mais on voit qu'au travers des cycles de vie, l'élevage peut être un pis-aller vers une autre activité comme une activité de repli en cas de non succès; vice versa l'activité non agricole peut être un moyen de capitaliser en bétail comme un tremplin vers un mode de vie citadin.

Suite aux sécheresses chroniques (1972, 1977, 1983-84, 1991-92) qui ont affecté les Peuls de la vallée du Sénégal, Santoir (1994) montre que si la pluriactivité a constitué une stratégie de survie, elle n'a pas induit de véritables ruptures sachant qu'elle a toujours fonctionné dans ce groupe social. «En 1975, on notait déjà que la moitié des ménages Peuls Sare de Matam et $21 \%$ des ménages Peuls Waalo de la basse vallée avaient recours à l'émigration pour compléter leurs ressources. Chez ces derniers, un ménage sur cing se consacrait au commerce (principalement petit bétail) et à la cueillette (gomme surtout) ; un ménage sur trois disposait de revenus tirés d'une activité extérieure au village : travail temporaire (tâcheron dans les périmètres ou à la Compagnie Sucrière Sénégalaise (CSS), Sardiennage des troupeaux villageois, charbon de bois, vente d'allumettes...)» (Santoir 1994). Dès lors, si la migration et le petit commerce prennent une place de plus en plus importante, on ne peut toutefois parler de reconversion. On peut aussi se demander, à l'instar de Sandron (1998), si cette pluriactivité spatiale dans les zones agropastorales dominées par des anciens nomades n'est pas une sorte de continuité dans la mobilité. «L'ex-nomade, s'il est maintenant fixé, bénéficie d'une culture séculaire du nomadisme qu'il ne sera pas facile d'évacuer en l'espace d'un décret» (Louis 1979, cité par Sandron 1998).

On voit bien que l'élevage est un moyen de gérer les crises ou difficultés dans le temps. Outre son apport de produits toute l'année à la différence des activités culturales, l'élevage permet de gérer des chocs climatiques et de favoriser les opportunités grâce à sa mobilité et à ses effets induits: transfert d'idées, changement social. Dans les zones agropastorales transfrontalières de l'Afrique de l'Est (Ethiopie, Kenya, Somalie) ou d'Afrique de l'Ouest (Mali, Niger, Burkina Faso), le commerce de bétail génère aussi une multiplicité d'activités parallèles (commerce, restauration, artisanat), qui peuvent être garantes d'un dynamisme régional; dans ces zones, en cas de perturbation du commerce de l'élevage, cette pluriactivité peut soit s'effondrer, soit, au contraire, jouer un rôle d'appoint. Ainsi, il est difficile de comprendre l'évolution de l'économie d'élevage sans intégrer ce dynamisme spatial et économique.

\section{4 / Mécanismes de gestion des risques induits par l'élevage}

Comme on l'a vu précédemment l'élevage présente en soi un potentiel formidable puisqu'il est capable de se développer dans des régions où toute autre activité économique semble compromise en raison des conditions naturelles. Mais, comme toute activité agricole, l'économie d'élevage est aussi soumise aux risques climatiques, agronomiques, sociaux, et économiques. A ces risques, se juxtaposent un ensemble de risques propres à l'activité d'élevage et qui sont en partie liés à la mobilité (risque de perte, risque de vol, risque de divagation, risque sanitaire plus élargi du fait des déplacements...). Dans les pays en développement, les exploitations, qui dépendent le plus de l'économie de l'élevage (pasteurs, agropasteurs), se situent souvent dans des zones difficiles, au climat peu propice à toutes activités culturales ce qui les rendent très dépendantes du marché pour leur approvisionnement en produits vivriers; alors que la diversification vers l'élevage est plus évidente dans les zones agricoles, en tenant compte bien évidemment de certaines contraintes comme la résistance aux trypanosomiases. Comme tout produit agricole, les produits animaux subissent les risques de méventes ou de mal vente (prix bas) ainsi qu'une détérioration des termes de l'échange (notamment avec les prix des céréales). A la différence de nombreux produits agricoles comme les céréales, les tubercules, les produits animaux sont très périssables et le stockage (à l'exception du stockage sur pied qui est très coûteux ou de la viande séchée traditionnelle) est limité dans les pays du Sud. Ceci induit une certaine rigidité de l'offre.

Face à ces risques, les producteurs ont su développer une multiplicité de stratégies anti-risque que l'on retrouve au travers des études sur les pratiques d'élevage. On peut citer :

1. Les faibles densités animales sur les pâturages ;

2. Les mobilités entre milieux complémentaires ;

3. Les troupeaux multi-espèces ayant des rythmes de reproduction, des besoins, des qualités différentes et complémentaires ;

4. La prédominance des femelles qui fournissent un potentiel de reproduction ;

5. La répartition sociale du cheptel entre les différentes unités d'exploitation (par prêts, héritages, dots) et à l'intérieur du ménage ;

6. Le faible taux d'exploitation du troupeau (vente, consommation) ;

7. Les échanges de biens et services (lait, mil, poisson, bétail) avec les sédentaires.

Ces mécanismes de gestion du risque s'inscrivent généralement dans le temps long pour les éleveurs. En Afrique du Nord, on a pu mettre en évidence un certain nombre de facteurs internes et externes aux exploitations qui expliquent l'abandon de ces modes de gestion des risques (Alary et El Mourid 2005). Parmi ces facteurs, prédominent les politiques de sédentarisation, puis les politiques de lutte contre les sécheresses (avec la distribution gratuite ou à faible prix de vastes stocks d'orge) qui se traduisent, aujourd'hui, par l'affaiblissement des mécanismes traditionnels de gestion des ressources pastorales favorisant les comportements opportunistes, individualistes au détriment du renouvellement des ressources et le maintien d'un cheptel surnuméraire en période de sécheresse par rapport à un stock de ressources naturelles affecté par les sécheresses répétitives. Le résultat est bien un épuisement des ressources pastorales et un accroissement de la dépendance des éleveurs visà-vis du marché des céréales (notamment l'orge en Afrique du Nord). Des dynamiques similaires de sédentarisation sont observées en Afrique subsaharienne en lien avec les sécheresses des dernières décennies et des perturbations politiques (Arditi 2009, Correra et al 2009). Cette sédentarisation s'accompagne d'une diversification croissante des activités et une activation plus rapide des multiples rôles de l'élevage. 


\section{4 / L'élevage dans la sécuri- té alimentaire et le dévelop- pement local}

L'élevage recouvre ainsi une pluralité de fonctions environnementales, sociales, culturelles et économiques. Nous avons vu que cette diversité de fonctions explique que l'élevage puisse jouer un rôle important dans les stratégies de gestion des risques et de réduction de la pauvreté des familles. Or, cette multifonctionnalité joue aussi un rôle primordial à l'échelle des régions, notamment dans la gestion de la sécurité alimentaire et le développement local.

\section{1 / Le rôle de l'élevage dans la sécurité alimentaire}

L'insécurité alimentaire constitue toujours un des problèmes les plus urgents, mais aussi les plus complexes auxquels doivent faire face la majorité des pays $\mathrm{du}$ Sud. On estime qu'environ 815 millions de personnes ont souffert de sous-nutrition et de malnutrition entre 1997 et 1999 (FAO 2001) ; ils seraient plus d'un milliard en 2009 (FAO 2009). Près de 987 millions de pauvres dans le monde dépendent de l'élevage pour leur moyen d'existence (Ashley et al 1999), et près de $70 \%$ des 1,4 milliard de personnes en extrême pauvreté (FAO 2009). D'après les travaux de Thornton et al (2003) sur la pauvreté et l'élevage dans le monde, le nombre d'éleveurs pauvres («poor livestock keepers») seraient de 397 millions, dont 162 millions en Afrique subsaharienne. Ainsi, l'élevage grâce à ses multiples produits et services constitue une activité essentielle de survie pour les ménages, notamment les sans-terre ou les petites exploitations (Upton 2004) mais aussi les femmes qui représentent $70 \%$ des pauvres dans le monde (DFID 2000). L'élevage joue aussi un rôle direct ou indirect fondamental dans la réduction de l'insécurité alimentaire à des échelles plus grandes que le ménage - qui peuvent être le village, un territoire ou une région plus large - en lien avec ses propres caractéristiques (notamment mobilité, cycles de production multiples).

Des études réalisées au Nord Mali (zone de Gao-Kidal à la frontière algérienne), en Afrique centrale ou dans la région de Somali en Ethiopie montrent comment les sociétés d'éleveurs ont su réguler leur approvisionnement en céréales et autres produits alimentaires par la constitution de réseaux sociopolitiques transfrontaliers très dynamiques basés sur les flux des animaux. Dans l'espace saharo-sahélien, les prin- cipaux convois de camions empruntent toujours les axes transsahariens des caravanes chamelières du début du $\mathrm{XX}^{\mathrm{e}}$ siècle (Grégoire et Schmitz 2000). Les échanges sont principalement construits sur des échanges anciens de marchandises, notamment les dattes contre du bétail (chameaux et moutons), du henné, du sésame et de l'arachide d'Afrique noire. Aujourd'hui l'Algérie inonde le marché africain de cartons de lait et d'autres denrées subventionnées et exportées en fraude en échange de bétail (ibid.). Ces flux transsahariens sont essentiellement clandestins afin de contourner les législations étatiques (notamment les mesures protectionnistes de l'Algérie ou la Libye), de ne pas acquitter de droits de douane et d'éviter les problèmes de change liés à la non convertibilité des monnaies comme le dinar algérien et libyen ou l'ouguiya mauritanien. Ils sont principalement contrôlés par les commerçants arabes, qu'ils soient algériens, libyens, maliens ou nigériens. Tout au long de leur parcours, ils ont installé, dans les principales localités, des petites communautés appartenant au même réseau social. $\mathrm{Ce}$ commerce clandestin est vital de part et d'autre de la frontière : il assure d'une part, l'approvisionnement de l'Algérie en ovins pour l'Aïd, fête musulmane pour laquelle l'Etat ne peut se permettre d'avoir des ruptures d'approvisionnement, et d'autre part, l'approvisionnement en denrées de première nécessité (lait, céréales, pâtes alimentaires...) des régions du Nord Mali, sans compter l'enjeu énergétique. Au cours d'interview dans la zone sur l'amélioration des conditions sanitaires des ani- maux, un chef traditionnel de la région de Gao demandait explicitement de ne pas perturber ce commerce au risque d'aggraver l'insécurité alimentaire de la zone (Alary et Nouhine Dieye 2006). On voit comment les priorités alimentaires passent avant les priorités sanitaires ou réglementaires. On retrouve aussi ces réseaux en Afrique centrale (Duteurtre et al 2002).

On retrouve ce dynamisme du commerce clandestin dans la zone pastorale entre l'Est éthiopien (Ogaden), la Somalie (Somaliland) et le Nord Kenya. Ce commerce est construit sur des liens ethniques ou claniques forts entre les trois zones. C'est ce réseau qui assure en partie l'approvisionnement des zones pastorales en produits de première nécessité comme les céréales (riz et blé), le sucre, l'huile, les dattes, etc. L'embargo sanitaire des pays du Golfe sur les ruminants en provenance de la zone, durant les crises sanitaires liées à la maladie de la Vallée du Rift au début des années 2000 , ont largement fragilisé la sécurité alimentaire de toute la zone (Pratt et al 2005). Les conséquences sont une détérioration des termes d'échange entre le bétail et les biens de consommation liée en partie à la baisse des prix des produits animaux qui s'est traduite par une réduction de 50 à $80 \%$ du commerce de produits de céréales; cela représente une réduction de 2 à 10 sacs de céréales par famille, affectant ainsi la sécurité alimentaires des ménages de la zone (ibid.).

Ces exemples montrent comment l'élevage participe à plusieurs dimensions spatiales des enjeux de sécurité

\section{Photo 3. Elevage laitier au Nigeria.}

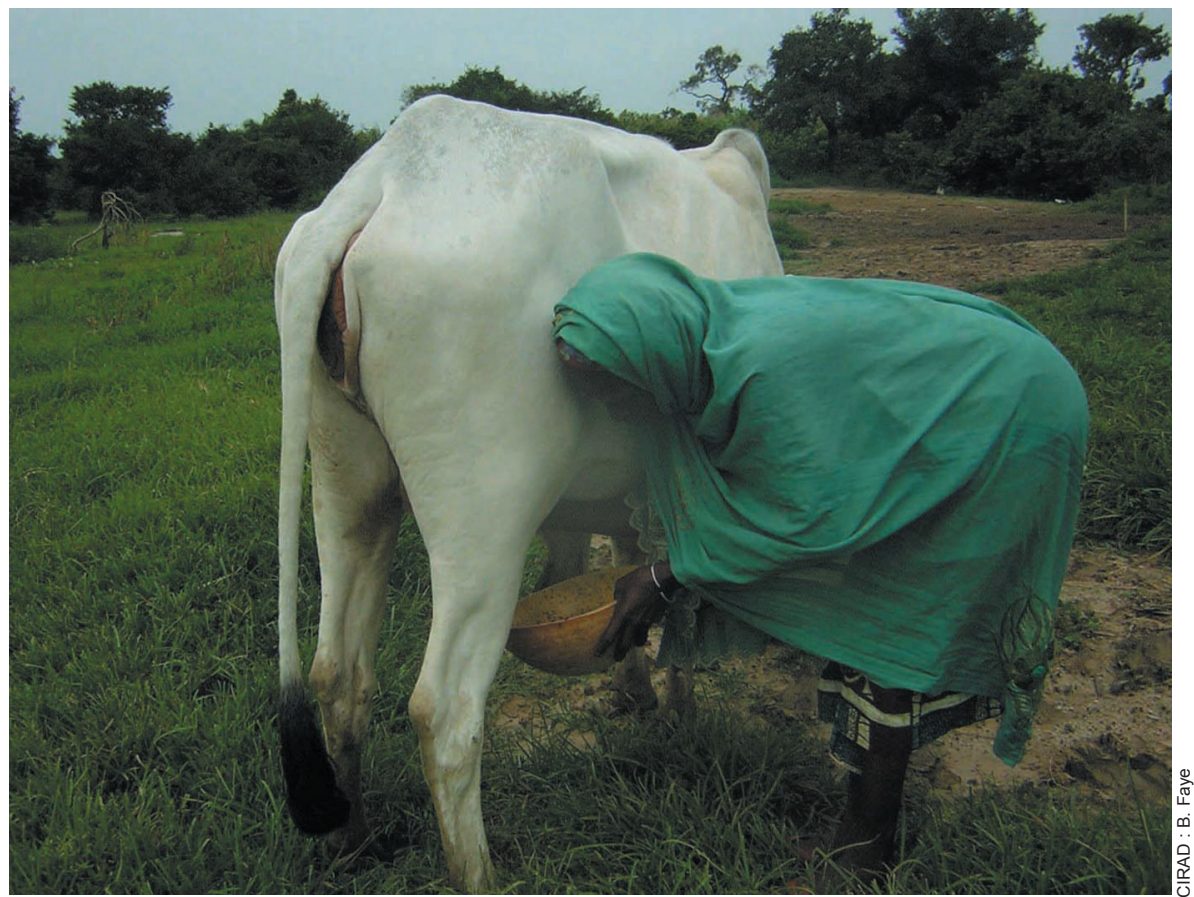


alimentaires. A cause de sa mobilité et sa valeur marchande, il participe à l'extension des réseaux marchands, notamment avec les zones agricoles pour l'approvisionnement des zones pastorales souvent incultes pour les produits agricoles de base. Facteur de migration, il a su favoriser le développement de multiples réseaux sociogéographiques qui sécurisent certains flux de marchandises et structurent de véritables filières de distribution des produits alimentaires. Ces filières sont principalement bâties sur des réseaux de confiance, des réseaux familiaux. Mais il faut noter que la nature des conventions détermine largement les termes des échanges et peuvent amener des distorsions des marchés.

L'élevage participe aussi à la dimension temporelle de la sécurité alimentaire. Il convient en effet de distinguer l'insécurité alimentaire conjoncturelle (qui peut être régulière ou inattendue) de l'insécurité alimentaire structurelle qui est souvent localisée géographiquement et/ou socialement et qui résulte d'un ensemble complexe d'éléments. Si la première fait appel à un système d'alerte précoce destinée à prévoir les crises et à déclencher les mécanismes de déstockage nationaux (redistribution des surplus) ou internationaux (aide alimentaire), la seconde s'inscrit davantage dans les dimensions sociales et politiques de la sécurité alimentaire. Selon Courade (1989), la question alimentaire doit être principalement vue comme un «fait social global», incluant un ensemble de régulations, d'arbitrages, de compromis ou conflits aux différentes échelles spatiales et temporelles. Ainsi, à l'échelle du ménage, l'appropriation par les femmes ou les cadets de certains animaux - permise par le système matrimonial ou social au sens large - permet de réguler certaines fonctions de sécurité alimentaire à l'échelle des ménages. Les femmes peuvent soigner le système d'alimentation de leur vache pour avoir plus de lait, vendre des animaux pour acheter des céréales ou produits alimentaires complémentaires pour le ménage, etc. Grâce aux cycles biologiques des animaux et la multiplicité des produits, les ménages accroissent leur capacité de sécurisation alimentaire à court terme par la production de lait ou de viande autoconsommée ou vendus, par la facilitation des déplacements et à moyen terme et long terme en favorisant les échanges marchands et donc les réseaux sociaux, en accroissant la productivité sur les terres (par la traction animale, la fumure), en créant aussi une capacité à demander un prêt en cas de coup dur. Ces réseaux sociaux bâtis sur les échanges d'animaux sont aussi soumis au risque de comportements des intermédiaires entre zones : ville-campagne, mais aussi zone rurale-zone d'élevage. Et certains comportements spécula- tifs ou opportunistes peuvent être à l'origine de crises alimentaires graves comme il est observé depuis un certain nombre d'années au Niger.

Ainsi l'élevage joue un rôle majeur dans la régulation de la sécurité alimentaire à différentes échelles de temps et d'espace. En outre, si l'élevage ruminant est principalement vu comme un produit d'échange dans les sociétés d'éleveur, la production de lait et sa transformation restent des activités majeures des ménages et participent à la fois à la satisfaction des besoins nutritifs du ménage (apport de protéines et vitamines), mais également à l'assurance des gestes traditionnels de convivialité. En Inde, près de $60 \%$ des bovins sont élevés par les producteurs marginaux et les petits producteurs dont la taille de l'exploitation est de 1,7 ha. Et plus des trois quarts de la production de lait en Inde sont autoconsommés (Barbier et al 2004). Les élevages de petits ruminants ou d'animaux à cycles courts (élevages porcin et avicole) participent plus facilement à la couverture des besoins alimentaires du ménage sans engager un processus de décapitalisation.

\section{2 / L'élevage dans les écono- mies locales}

On estime que dans l'ensemble des pays en développement, $60 \%$ des ménages possèdent des animaux (FAO 2009). L'agriculture en général et l'élevage en particulier fourniraient $52,5 \%$ des emplois sur l'ensemble de l'Afrique de l'Ouest (CSAO 2007). A la fin des années 90 , le système coopératif indien, NDDB, compte plus de 9,7 millions de producteurs, membres de 75000 sociétés villageoises qui assurent la réception du lait et le paiement journalier des producteurs. Ces sociétés sont affiliées à 170 Unions au niveau du district, rattachées elles-mêmes à 22 Fédérations au niveau des états. Les Unions assurent la collecte du produit et son conditionnement, ainsi que l'approvisionnement des villes au niveau du district. Près d'un tiers des Unions ont investi dans une unité de fabrique d'aliments concentrés pour bétail. La Fédération se charge de la commercialisation du produit entre les districts et les états, mais aussi de la gestion des aides financières et du secteur recherche/ développement dans le domaine du lait et de l'élevage. Les sociétés villageoises développent aussi des centres de santé, des écoles, etc. En outre, l'opération Flood a permis la valorisation d'autres activités agricoles (tabac et canne à sucre dans le Gujarat) grâce au désenclavement des villages. Ainsi, l'élevage a réussi à lever en partie la contrainte foncière à l'échelle des villages pour répondre à un défi national.
Si ce type d'expérience fait exception, des recherches récentes menées au Sénégal, au Mali et en Ethiopie dans le cadre du projet Icare ont analysé les effets de l'ouverture récente des marchés de produits animaux sur le développement local et plus particulièrement sur le développement de zones d'élevage. L'analyse s'est appuyée entre autres sur un éclairage croisé de l'économie, de la sociologie et de la géographie pour comprendre la «construction sociale» des liens marchands, un facteur important du développement territorial (Duteurtre 2010). Ces travaux montrent entre autres une renégociation permanente des éleveurs vis-à-vis du marché, des choix institutionnels qui sont opérés - susceptibles de changer selon le jeu des pouvoirs et influences -, de dynamiques collectives d'appropriation des ressources à l'échelle des territoires. Si l'élevage est un facteur moteur des dynamiques territoriales dans un certain nombre de zones agricoles en favorisant l'emploi, les transactions interrégionales, voire internationales, la plupart des mécanismes de régulation dépendent de la pérennité des relations sociales, ellemême sensible aux jeux sociopolitiques.

Le Millenium ecosystem assessment offre un cadre de prise en compte des fonctions environnementales de l'élevage et de l'agriculture, à travers la reconnaissance des services écosystémiques à l'échelle locale. Cette approche est notamment mobilisée pour défendre l'idée que ces services pouvaient être internalisés, c'est-à-dire qu'il serait possible de mettre en place des procédures de paiement pour services environnementaux par des mécanismes marchands. Cependant, ces types de paiement sont restés jusqu'à présent peu mis en œuvre dans le secteur de l'élevage. Aux niveaux nationaux et locaux, les politiques sectorielles d'élevage ont jusqu'à présent peu pris en compte les fonctions non économiques de l'élevage. Et les grands projets d'appui à l'élevage continuent de considérer les animaux comme des moyens de production qu'il s'agit de sécuriser en vue d'améliorer la production qui en est tirée (Duteurtre et Faye 2009). D'importants programmes sanitaires, hydrauliques, ou zootechniques, ou même de nombreux projets de microcrédit d'appui à l'élevage (crédits «vaches laitières», projets tels que «zébunet»...) ont été ainsi mis en place dans cette optique essentiellement économique.

$\mathrm{Au}$ Burkina Faso, l'analyse de la contribution de l'élevage dans la réduction de la vulnérabilité montre par estimation que 60 à 90000 personnes seraient directement concernées dans les filières animales sans inclure les emplois indirects: gardiens, restaurateurs sur les marchés, vendeurs 
d'aliments ou autres produits pour bétail, vétérinaires, etc. (IEPC 2001). Mais la vulnérabilité de ces petits métiers est évidemment particulièrement forte et les filières de commercialisation des produits animaux représentent un autre aspect de l'élevage qu'une stratégie de lutte contre la pauvreté doit prendre en compte et qui est totalement indépendante de la productivité des systèmes, des cheptels ou des terroirs.

Plus généralement, l'élevage est peu pris en compte dans les politiques de réduction de la pauvreté (réputé parfois comme capital pour riches) alors qu'il est souvent le seul moyen de survie, soit dans les zones agroclimatiques difficiles, soit les zones densément peuplées où l'accès au foncier est devenu trop coûteux, voire impossible au vu du morcellement foncier. Blench et al (2003) montrent qu'il y a très peu de lien entre l'importance de l'élevage dans un pays et sa prise en compte dans les Documents de politique et Stratégies de Réduction de la Pauvreté (DSRP). Seulement quatre pays d'Afrique (Guinée, Mauritanie, Mozambique et Rwanda) ont proposé une stratégie propre à l'élevage (ibid.). Toutefois, certains pays comme le Kenya, le Soudan, l'Ouganda ou le Burkina Faso ont proposé une stratégie de développement de l'élevage en tenant compte de la pauvreté (PicaCiamarra 2005). D'autres ont pris en compte l'élevage dans les politiques nationales comme en Ethiopie ou au Mali (ibid.).

\section{Conclusion}

Dans les sociétés d'éleveurs des zones difficiles, notamment les zones pastorales d'Afrique subsaharienne et Afrique du Nord ou les zones montagneuses d'Amérique latine, l'élevage est au cœur du système de régulation, à la fois de l'espace, mais aussi des formes d'alliance (comme les règles matrimoniales), des règles d'échange (rapports économiques : élevage/céréales), ou des mécanismes de reproduction générationnelle. La mobilité incarnée dans le modèle nomade est souvent le marqueur d'appartenance culturelle à une société d'éleveur fonctionnant sur un système de valeur entièrement ancrée dans le système d'élevage.

Si les conditions du milieu expliquent fortement la répartition des systèmes d'élevage selon les espèces dominantes et le mode de gestion de l'espace et des ressources (mobilité permanente ou temporaire...), on peut noter une large extension de l'élevage chez les cultivateurs, les sans-terre, voire les salariés des zones urbaines et périurbaines. Du complément de trésorerie à la constitution d'un capital, cet élevage modifie profondément le paysage environnemental et socio-économique.

Un des grands enjeux du développement de l'élevage est bien en lien avec la croissance démographique qui se traduit par différentes conséquences : une plus grande demande de produits alimentaires, une pression accrue sur le foncier agricole, mais aussi non agricole que l'on retrouve à la fois dans les zones arides et les zones urbaines. Or l'élevage à la différence de la production culturale peut se développer sans foncier ou presque... Si l'élevage a besoin du foncier pour son alimentation, il est capable de se développer dans les zones urbaines et périurbaines en valorisant les sous-produits humains et facilitant le transport de biomasse entre la ville et la campagne. Cette capacité explique que près de 1 million de ménages sans terre ont développé une activité d'élevage (FAO 2009). Cette activité est très prisée des femmes qui peuvent la conduire depuis leur domicile (DFID 2000). En revanche, dans les zones difficiles, les effets de la croissance démographique se traduisent souvent par une menace de désertification sur les zones pastorales aggravant les phénomènes de migration et d'urbanisation.

De plus, si l'élevage constitue un moyen de sortie de la pauvreté pour les sans-terre ou un instrument d'épargne pour les plus aisés ou encore une sécurité pour la majorité, il est aussi soumis à un ensemble de risques, notamment en lien avec le changement climatique (Narbone et al 2010). Ainsi, face à la croissance de la demande en viande et lait, l'élevage a un rôle majeur à jouer dans les politiques de sécurité alimentaire bien que son devenir va dépendre des mécanismes de son intégration spatiale et temporelle dans les zones agricoles, des formes d'intégration avec les autres activités (gestion de la fertilité, effet sur l'environnement) et des systèmes d'assurance dans les logiques d'adaptation pour dépasser le stade de stratégie de survie (coping stratégie).

\section{Références}

Adjamagbo A., 1997. Les solidarités familiales dans les sociétés d'économie de plantation. Le cas de la région de Sassandra en Côte d'Ivoire. In: Ménages et familles en Afrique. Approches des dynamiques contemporaines. Pilon M., Locoh T., Vignikin E., Vimard P. (Eds). Les études du CEPED, IRD, Paris, France, 15, 301-325.

Alary V., 1999. Le système coopératif laitier en Inde face à la libéralisation. Econ. Rurale, 252, 35-41.

Alary V., 2009. Le rôle de l'élevage laitier dans la gestion des risques paysans : diagnostic et modélisation des exploitations agricoles du nord de l'Inde. In : L'élevage, richesse des pauvres. Duteurtre G., Faye B. (Eds). Editions Quae, Versailles, France, 163-178.

Alary V., El Mourid M., 2005. Les politiques alimentaires au Maghreb et leurs conséquences sur les sociétés agropastorales. Rev. Tiers Monde, 65, 785-810.

Alary V., Nouhine Dieye, P., 2006. Etude du commerce régional de bétail entre le Mali et l'Algérie. Projet STDF 13 (Standard and Trade Development Facilities), FAO/OIE, Mission Mali du 11 au 17 décembre 2006, Rapport de mission, CIRAD, 2006, Montpellier, France, 31p.

Alary V., Chalimbaud J., Faye B, 2007. Multiple determinants of milk production in Africa, example of the diversity of dairy farming systems in Mbarara area (Uganda). Afr. Dev., 32, 156-180.

Anderson D.M., 1999. Rehabilitation, resettlement and restocking: ideology and practice in pastoralist development. In: The poor are not us. Poverty and pastoralism. Anderson D.M. Broch-Due V. (Eds). Publ. Eastern African Studies, Oxford, UK, 240-256.

Arditi C., 2009. La paupérisation des éleveurs Peuls du RCA. In : L'élevage, richesse des pauvres. Duteurtre G., Faye B. (Eds). Editions Quae, Versailles, France, 37-49.

Ashley S., Holden S., Bazeley P., 1999. Livestock in poverty-focused development crewkerne: livestock in development. http: /www.smallstock.info/reference/LID/livestock.pdf

Aubron C., 2007. Lait et fromage dans un pays andin : quelle place pour les filières arti- sanales péruviennes face aux industries laitières ? In : Numéro spécial Filière lait. Rev. Elev. Méd. Vét. Pays Trop., 189-197.

Auclair L., Gubry P., Picouet M., Sandron F., 2001. Régulations démographiques et environnement. IRD-LPE-CEPED. Etudes du CEPED, Paris, France, 19, 284p.

Barbier B., Alary V., Deybe D., 2004. L'agriculture et l'élevage dans les plaines indogangétiques de l'Inde : vers une nouvelle intégration. OCL, Oléagineux Corps Gras Lipides, 11, 277-286.

Bernus E., 1975. Les composantes géographiques et sociales des types d'élevage en milieu Touareg. In : Pastoralims in Tropical Africa. Monod T. (Ed). International African Institute, Oxford University Press, UK, 229-244.

Bernus E., 1981. Touaregs nigériens, unité culturelle et diversité régionale d'un peuple pasteur. Mémoires ORSTOM, 94, 519p.

Bernus E., 1984. The Tuareg. Wanderers of the desert. Swissair Gazette, 20-28. http://www.documentation.ird.fr/hor/fdi:21205 
Bittencourt Machado G., 2009. Multi-fonctionnalité de l'agriculture familiale et diversification des activités dans le Sertao semi-aride de l'Etat de Bahia (Brésil). Thèse de doctorat, spécialité agriculture comparée, AgroParisTech, Paris, France, $305 p+$ annexes.

Blench R., Chapman R., Slaymaker T., 2003. A Study of the role of livestock in poverty reduction strategy Papers. PPLPI Working Paper, FAO, Rome, Italy, 1, 52p. www.fao. org/AG/againfo/programmes/en/pplpi/.../execsumm_wp01.pdf

Bommel P., Bonaudo T., Barbosa T., Bastos da Veiga J., Vieira Pak M., Tourrand J.F., 2010. La relation complexe entre élevage et forêt en Amazonie brésilienne : une approche par la modélisation multi-agents. Cah. Agric., 19, 104-111.

Bonfiglioli A.M., 1990. Pastoralisme, agropastoralisme et retour: itinéraires sahéliens, Cah. Sci. Hum., 26, 255266.

Bonte P. 2006. Anthropologies des sociétés nomades. Fondements matériels et symboliques (première partie). Département de sociologie, Mineure d'anthropologie, Second semestre 2006. www.univ-paris8.fr/sociologie/fichiers/bonte-cours.pdf

Bonte P., 2007. Essai sur les formations tribales du Sahara occidental. Approches comparatives, anthropologiques et historiques. Pire L. (Ed). Liège, Belgique, 190p.

Bonte P., 2008. L'émirat de l'Adrar mauritanien, Harîm, compétition et protection dans une société tribale saharienne. Karthala, Collection Hommes et Sociétés, Paris, France, 576p.

Boutrais J., 1994. Les Foulbé de l'Adamaoua et l'élevage : de l'idéologie pastorale à la pluriactivité. Cah. Etudes Afr., 34, 175-196.

Bravo-Baumann H., 2000. Capitalisation of experiences on the contribution of livestock projects to gender Issues. Working document. Swiss Agency for Development and Cooperation. Bern, Sept. 2000, 30p. http://www.fao.org/WAIRDOCS/LEAD/X610 $\underline{6 \mathrm{E} / \mathrm{x} 6106 \mathrm{e} 01 . \mathrm{htm}}$

Brisebarre A.M., Kuczynski L., 2009. La Tabaski au Sénégal, Une fête musulmane en milieu urbain. Karthala, Paris, France, 468p.

Carter M., Barrett C., 2006. The economics of poverty traps and persistent poverty: an assetbased approach. J. Dev. Studies, 42, 178-199.

Coleman J., 1988. Social capital in the creation of human capital. Am. J. Sociol., 94, 95-120.

Collectif groupe Polanyi, 2008. La multifonctionnalité de l'agriculture : une dialectique entre marché et identité. Editions Quae, Versailles, France, 349p.

Corniaux C., Le Mercier J., Diallo A., 1999. Utilisation des sous produits agricoles et agroindustriels dans les élevages bovins du Delta du fleuve Sénégal. Bull. Inf., PSISénégal/SAED, St Louis, Sénégal, 3, 11p.

Corniaux C., Alary V., Cloarec M., 2009. Poids de l'élevage dans l'économie monétaire des ménages et vulnérabilité des agro-éleveurs en zone Office du Niger (Mali). Renc. Rech. Rum., Paris, France. http://www.instelevage.asso.fr/3R-new/spip.php?article2938.

Correra A., Lefeuvre J.C., Faye B., 2009. Organisation spatiale et stratégie d'adaptation des nomades du Parc National du Banc d'Arguin à la sécheresse. Rev. Sècheresse, 19, 245-251.
Courade G., 1989. Le risque d'insécurité alimentaire: de l'imprudence écologique au démantèlement de l'Etat providence. In : Le risque en agriculture. Eldin M., Milleville P. (Eds). Ed. ORSTOM, Coll. A travers champs, Paris, France, 575-597.

CSAO, 2007. L'avenir de l'élevage au Sahel et en Afrique de l'Ouest: Potentialités et défis dans la perspective d'un renforcement du marché régional. Secrétariat du Club du Sahel et de l'Afrique de l'Ouest (CSAO), Paris, France, 145p.

Delgado C., Rosegrant M., Steinfeld H., Ehui S, Courbois C., 1999. Livestock to 2020: The Next food revolution 2020. Washington, DC: International Food Policy Research Institute (IFPRI).

DFID, 2000. Halving world poverty by 2015, economic growth, equity and security: Strategies for achieving the international development targets. DFID Strategy Paper, 54p. http://www.dfid.gov.uk/Pubs/files/tsp economic.pdf

Dupire M., 1970. Organisations sociales des Peuls. Plon, Paris, France, 624p.

Duteurtre G., 2003. La typicité du beurre de vache en Ethiopie. Études et Recherches Sahéliennes, 8-9, 59-66.

Duteurtre G., 2010. Accès aux marchés et développement des élevages en Afrique : la construction sociale des liens marchands. 4èmes Journ. SFER-INRA-CIRAD «Sciences sociales», Agrocampus Rennes, 9 et 10 décembre 2010, 14p.

Duteurtre G., Faye B., 2009. L'élevage, richesse des pauvres. Collection Update Sciences \& Technologies, Editions Quae, Versailles, France, 284p.

Duteurtre G., Koussou M.O., Essang T., Kaderoy-Tiguague D., 2002. Le commerce de bétail dans les savanes d'Afrique centrale : réalités et perspectives. In : Savances africaines : des espaces en mutation, des acteurs face à de nouveaux défís. Jamin J.Y., Seiny Boukar L. (Eds). Actes du colloque, mai 2002, Garoua, Cameroun, N'Djamena, Tchad, Prasac, 8p.

Dutilly-Diané C., Alary V., Bendaoud M., 2005. Modèle Ait Ammar : la modélisation du parcours collectif. In : Actes de l'atelier final du projet FEMISE (FEM2-02-21-05). Les obstacles aux transferts technologiques dans les petites et moyennes exploitations des zones arides et semi arides du Maghreb. Mekersi S., Alary V., Cherfaoui M.L. (Eds). FEMISE/ICARDA/ INRAA/ CIRAD, 6-7 déc. 2004, Alger, Algérie, 223-244.

El Aich A., Waterhouse A., 1998. Small ruminants in environmental conservation. Small Rum. Res., 34, 271-287.

Ellis F., Mdoe N., 2003. Livelihoods and rural poverty reduction in Tanzania. World Dev., 31, 1367-1384.

Fafchamps M., Minten B., 2002. Returns to social network capital among traders. Oxford Econ. Papers, 54, 173-206.

FAO 2001. The state of food insecurity in the world 2001. Economic and social department. http://www.fao.org/docrep/003/y1500e/ $\mathrm{y} 1500 \mathrm{e} 00 . \mathrm{htm}$

FAO 2009. The State of Food and Agriculture 2009 : Livestock in the Balance, FAO, Rome, Italie, 166p. http://www.fao.org/ docrep/012/i0680e/i0680e00.htm

Faye B., 1992. L'élevage et les éleveurs de dromadaires dans la Corne de l'Afrique. In :
Relations Homme-animal dans les sociétés pastorales d'hier et d'aujourd'hui. Festival animalier International de Rambouillet. 2526 sept., Actes du Colloque, 59-72.

Faye B., 2009. Pauvreté et solidarité chez les peuples pastoraux. In : L'élevage, richesse des pauvres. Duteurtre G., Faye B. (Eds). Editions Quae, Versailles, France, 77-87.

Gallais J., 1989. Une géographie politique de l'Ethiopie. Le poids de l'Etat. Economica, Paris, France, 213p.

Gibon A., 1997. Addressing livestock farming systems ecological sustainability at the regional level: an example from the Central Pyrenees. In: Livestock farming systems: More than food production. Sørensen J.T. (Ed), EAAP Publ., 89, 30-41.

Grégoire E., Schmitz J., 2000. Monde arabe et Afrique noire: permanences et nouveaux liens. Autrepart, 16, 5-20.

Ickowicz A., Bah A., Bommel P., Choisis J.P., Etienne M., Gibon A., Lasseur J., Morales H., Touré I., Tourrand J.F., 2010. Facteurs de transformation des systèmes d'élevage extensifs des territoires: étude comparée des dynamiques locales sur trois continents. Cah. Agric., 19, 127-134.

IEPC, 2001. Burkina Faso : Initiative Elevage, pauvreté et croissance (IEPC). Propositions pour un Document national. Annexe 7 : Commercialisation des produits et intrants de l'élevage. FAO/Banque Mondiale/ groupe IEPC Mali, 61p.

Lesourd M., 1997. L'archipel rural africain en mouvement. In : La ruralité dans les pays du Sud à la fin du vingtième siècle. Gastellu J.M. (Ed). ORSTOM, Paris, France, 363-378.

Levi-Strauss C., 1967. Les structures élémentaires de la parenté. Edition Mouton \& Co., Paris, France, 218p.

Mahieu F.R., 1989. Transferts et communauté africaine. Statéco, 58-59, 107-136.

Narbone A., Ronchi B., Lacetera N., Ranieri M.S., Bernabucci U., 2010. Effects of climate changes on animal production and sustainability of livestock systems. Livest. Sci., 130, 57-69.

Nefzaoui A., El Mourid M., 2008. Rangeland improvement and management in arid and semi-arid environments of West Asia and North Africa. CRDI/FIDA, 20p. www.mashreq-maghreb.org/.../KariaNetPublicationonRangelandsImprovementen2. pdf

Ninot O., 2010. Des moutons pour la fête : l'approvisionnement de Dakar en moutons de Tabaski. Cah. Outre-Mer, 1, 249, 141-164.

Nordblom T.L., Goodchild A.V., Shomo F., Gintzburger G., 1997. Dynamics of feed resources in mixed farming systems of the North Africa, West and Central Asia regions. In: Crop residues in sustainable mixed crops/livestock farming systems. Renard C. (Ed). CAB International, London, UK, 131-148.

Pica-Ciamarra U., 2005. livestock policies for poverty alleviation: theory and practical evidence from Africa, Asia and Latin America. PPLPI Working paper, FAO, Rome, Italie, 27.

Pratt A.N., Bonnet P., Jabbar M., Ehui S., de Haan C., 2005. Benefits and Costs of compliance of sanitary regulations in livestock 
markets: the case of Rift Calley Fever in the Somali region of Ethiopia, Nairobi, Kenya. International Livestock Research Institute, The World Bank, 70p.

Putnam R.D., Leonardi R., Nanetti R.Y. 1993. Making democracy work: civic traditions in modern Italy. Princeton University Press, Princeton, 280p.

Reardon T., Delgado C., Matlon P., 1992. Determinants and effects of income diversification, amongst farm households in Burkina Faso. J. Dev. Stud., 28, 264-296.

Requier-Desjardins D., 2003. Le capital social dans la théorie économique : actif privé ou bien public ? Le point sur quelques contributions récentes. In : Regards croisés sur le capital social. Ballet J., Guillon R. (Eds). L'Harmattan, Coll. Ethique Economique, Paris, France, 15-39.

Rutagwenda T., Lechner-Doll M., Kaske M., Engelhardt W.V., Schultka W., Schwartz H.J, 1989. Adaptation strategies of camels on a thornbush savannah pasture, comparison with other domestic animals. CIHEAM, Options Méd., Série Séminaires, 2, 69-73.

Sandron F., 1998. L'immobilité forcée : la sédentarisation des nomades dans le Sud tunisien. Autrepart, 5, 63-78.

Santoir C., 1994. Décadence et résistance du pastoralisme. Les peuls de la Vallée du fleuve
Sénégal. Cah. d'Etudes Afr., 34, 133-135, 231 263.

Sen A., 1992. Inequality reexamined. London, Oxford University Press, UK, 207p.

Sen A., 2002. Ethique et économie. PUF, Quadrige, 364p.

Sow-Dia F., Somda J., Kamuanga M., 2007. Dynamique des filières laitières en zone sahélienne: cas de l'offre et de la demande du lai en zone agropastorale centre du Sénégal. In : Numéro spécial Filière lait. Rev. Elev. Méd. Vét. Pays Trop., 77-88.

Steinfeld H., Gerber P., Wassenaar T., Caste V., Rosales M., de Haan C., 2006. Livestock's long shadow: environmental issues and options. FAO, Rome, Italie, 391p.

Steinfeld H., Mooney H.A., Scheider F, Neville L.E., 2010. Livestock in a changing landscape Volume 1: Drivers, Consequences and Responses. IslandPress, Washington, USA, 1, 396p.

Tchayanov A., 1924. L'organisation de l'économie paysanne, Traduction d'Alexis Berelowitch, Ed. Maison des Sciences de l'Homme, Paris, France, 342p.

Thébaud B., 1988. Elevage et développement au Niger. Bureau International du Travail, Genève, Suisse, $82 p$.

Thornton P.K., Kruska R L., Henninger N., Kristjanson P M., Reid R.S., Robinson T P.,
2003. Locating poor livestock keepers at the global level for research and development targeting. Land Use Policy, 20, 311322.

Tichit M., Hubert B., Doyen L., Genin D., 2004. A viability model to assess the sustainability of mixed herds under climatic uncertainty. Anim. Res., 53, 405-417.

Tourrand J.F., 2009. La vache, richesse des migrants en agriculture familiale de l'Amazonie brésilienne. In : L'élevage, richesse des pauvres. Duteurtre G., Faye B. (Eds). Editions Quae, Versailles, France, 179190.

Upton M., 2004. The role of livestock in economic development and poverty reduction. PPLPI FAO, Roma, Italy. Working paper, 10. http://www.fao.org/ag/againfo/programmes/en /pplpi/docarc/abst10.html

Waller R.D., 1999. Pastoral poverty in historical perspective. In: the poor are not us. poverty and pastoralim in eastern Africa. Anderson D.M., Broch-Die V. (Eds). Coll. Eastern African Studies, James Currey, Oxford, UK, 20-49.

Woolcock M., Narayan D., 2000. Social capital: implications for development theory, research and policy. World Bank Res. Observer, $15,225-249$

\section{Résumé}

Par sa faculté à se développer dans des espaces restreints ou dans des milieux agro-écologiques difficiles, l'élevage constitue une opportunité pour une très grande diversité de groupes sociaux dans presque tous les milieux géographiques. Objet de nombreux échanges économiques, sous forme de troc ou d'échanges monétaires, l'élevage génère aussi des prestations sociales sous forme de dons, d'héritages ou de confiages. L'activité d'élevage permet ainsi dans de nombreux contextes la structuration de réseaux sociaux qui participent à la solidarité et à la sécurité des groupes humains, et constitue un facteur important de renforcement des actifs économiques et du capital social des familles. L'élevage est ainsi un objet complexe aux rôles multiples qui constituent autant d'atouts ou d'opportunités pour les éleveurs. Mais cette multifonctionnalité explique aussi que l'élevage soit au cœur de nombreux enjeux de développement pour les régions, à l'interface entre les objectifs de sécurité alimentaire et les objectifs de développement humain, environnemental et sociétal. Le rôle de l'élevage est ainsi l'objet de controverses qui s'expriment notamment au niveau des familles d'éleveurs, mais aussi au niveau des économies locales et nationales. Cette revue du rôle de l'élevage dans les sociétés du Sud montre en quoi l'élevage constitue un moyen de lutte contre la pauvreté dans des milieux agro-écologiques ou sociaux contraints sans pour autant négliger les risques inhérents à toute activité du vivant en interaction avec son milieu.

\section{Abstract}

\section{Livestock and societies: multiple roles of livestock in tropical countries}

Due to its ability to develop in confined spaces or in harsh agro-ecological environments, livestock is important for a wide variety of social groups in almost all geographical locations. Livestock is part of many economic exchanges, in the form of barter or monetary exchange. Livestock also generates social benefits in the form of gifts, inheritances or "foster care". Livestock enables the structuring of social networks that contribute to the solidarity and security of human groups and it is an important factor in strengthening the economic and social capital of families. Livestock is then a complex object with multiple roles that are essential assets or opportunities for farmers. But this multi-functionality also explains that livestock is at the heart of many development issues for regions, at the interface between food security goals and objectives of human, environmental and societal development,. This review on the roles of livestock in southern societies shows how livestock is a way to fight against poverty in harsh agro-ecological environments or in zones with strong social constraints without neglecting the risks inherent to any activity in interaction with the social and ecological environment.

ALARY V., DUTEURTRE G., FAYE B., 2011. Élevages et sociétés : les rôles multiples de l'élevage dans les pays tropicaux. In : Numéro spécial, Elevage en régions chaudes. Coulon J.B., Lecomte P., Boval M., Perez J.M. (Eds). INRA Prod. Anim., 24, 145-156. 